Access to this work was provided by the University of Maryland, Baltimore County (UMBC) ScholarWorks@UMBC digital repository on the Maryland Shared Open Access (MD-SOAR) platform.

Please provide feedback

Please support the ScholarWorks@UMBC repository by emailing scholarworks-group@umbc.edu and telling us what having access to this work means to you and why it's important to you. Thank you. 


\title{
On Adaptive-Multilevel BDDC
}

\author{
Bedřich Sousedík ${ }^{1,2} *$ and Jan Mandel ${ }^{1} \dagger$ \\ 1 Department of Mathematical and Statistical Sciences, \\ University of Colorado Denver, Campus Box 170, Denver, CO 80217, USA \\ 2 Institute of Thermomechanics, Academy of Sciences of the Czech Republic, \\ Dolejškova 1402/5, 18200 Prague 8, Czech Republic \\ jan.mandel@ucdenver.edu, bedrich.sousedik@ucdenver.edu
}

\section{Introduction}

The BDDC method (Dohrmann [2003]) is one of the most advanced methods of iterative substructuring. In the case of many substructures, solving the coarse problem exactly becomes a bottleneck. Since the coarse problem has the same structure as the original problem, it is straightforward to apply the method recursively to solve it only approximately. The two-level BDDC analysis has been extended into three-levels in a pioneering work by Tu [2007a,b], and into a general multilevel method by Mandel et al. [2008]. The methods for the adaptive selection of constraints for the two-level BDDC method have been studied in Mandel and Sousedík [2007a], Mandel et al. [2009]. Here we combine the two approaches into a new method preserving parallel scalability with increasing number of subdomains and excellent convergence properties.

The theoretical aspects of the design of the BDDC and a closely related FETI-DP on irregular subdomains in the plane has been studied by Klawonn et al. [2008]. The authors in particular demonstrated that a proper choice of a certain scaling can significantly improve convergence of the methods. Our goal here is different. We consider only the standard stiffness scaling and we look for a space where the action of the BDDC preconditioner is defined. A combination of these two approaches, also with the proper choice of initial constraints (Burda et al. [2009]), would be of independent interest. The presented algorithm has been recently extended into 3D in Sousedík [2010].

Klawonn and Rheinbach [2007, 2008] have recently successfully developed and extensively used several inexact solvers for the FETI-DP method, and Tu [2008] has extended the three-level BDDC for the saddle point problems.

All abstract spaces in this paper are finite dimensional. The dual space of a linear space $U$ is denoted by $U^{\prime}$, and $\langle\cdot, \cdot\rangle$ is the duality pairing.

\footnotetext{
* Partially supported by National Science Foundation under grant DMS-0713876 and by the Grant Agency of the Czech Republic under grant 106/08/0403.

$\dagger$ Supported by National Science Foundation under grant DMS-0713876.
} 


\section{Abstract BDDC for a Model Problem}

Let $\Omega \subset \mathbb{R}^{2}$ be a bounded polygonal domain, decomposed into $N$ nonoverlapping polygonal substructures $\Omega_{i}, i=1, \ldots, N$, which form a conforming triangulation. That is, if two substructures have a nonempty intersection, then the intersection is a vertex, or a whole edge. Substructure vertices will also be called corners. Let $W_{i}$ be the space of Lagrangean $P 1$ or $Q 1$ finite element functions with characteristic mesh size $h$ on $\Omega_{i}$, and which are zero on the boundary $\partial \Omega$. Suppose that the nodes of the finite elements coincide on edges common to two substructures. Let

$$
W=W_{1} \times \cdots \times W_{N},
$$

and let $U \subset W$ be the subspace of functions that are continuous across the substructure interfaces. We wish to solve the abstract linear problem

$$
u \in U: a(u, v)=\langle f, v\rangle, \quad \forall v \in U,
$$

for a given $f \in U^{\prime}$, where $a$ is a symmetric positive semidefinite bilinear form on some space $W \supset U$ and positive definite on $U$. The form $a(\cdot, \cdot)$ is called the energy inner product, the value of the quadratic form $a(u, u)$ is called the energy of $u$, and the norm $\|u\|_{a}=a(u, u)^{1 / 2}$ is called the energy norm. The operator $A: U \mapsto U^{\prime}$ associated with $a$ is defined by

$$
a(u, v)=\langle A u, v\rangle, \quad \forall u, v \in U .
$$

The values of functions from $W$ at the corners and certain averages over the edges will be called the coarse degrees of freedom. Let $\widetilde{W} \subset W$ be the space of all functions such that the values of any coarse degrees of freedom have a common value for all relevant substructures and vanish on $\partial \Omega$. Define $U_{I} \subset U \subset W$ as the subspace of all functions that are zero on all substructure boundaries $\partial \Omega_{i}, \widetilde{W}_{\Delta} \subset W$ as the subspace of all function such that their coarse degrees of freedom vanish, $\widetilde{W}_{\Pi}$ as the subspace of all functions such that their coarse degrees of freedom between adjacent substructures coincide and such that their energy is minimal. Then

$$
\widetilde{W}=\widetilde{W}_{\Delta} \oplus \widetilde{W}_{\Pi}, \quad \widetilde{W}_{\Delta} \perp_{a} \widetilde{W}_{\Pi} .
$$

The component of the BDDC preconditioner computed in $\widetilde{W}_{\Pi}$ is called the coarse problem, cf. [Mandel et al., 2008, Algorithm 11]. Functions that are $a$-orthogonal to $U_{I}$ are called discrete harmonic. In Mandel et al. [2005] and Mandel and Sousedík [2007a], the analysis was done in spaces of discrete harmonic functions after eliminating $U_{I}$; this is not the case here, so $\widetilde{W}$ does not consist of discrete harmonic functions only. Denote by $P$ the energy orthogonal projection from $U$ to $U_{I}$. Then $I-P$ is known as the projection onto the discrete harmonic functions. Finally, let $E$ be a projection from $\widetilde{W}$ onto $U$ defined by taking some weighted average over the substructure interfaces. 
Let us briefly describe the construction of the space $\widetilde{W}$ using the coarse degrees of freedom. Suppose we are given a space $X$ and a linear operator $C: W \mapsto X$ and define

$$
\widetilde{W}=\{w \in W: C(I-E) w=0\} .
$$

The values $C w$ will be called the local coarse degrees of freedom. To represent their common values, i.e. the global coarse degrees of freedom, suppose there is a space $U_{c}$ and linear operators

$$
Q_{P}^{T}: U \rightarrow U_{c} \quad R_{c}: U_{c} \rightarrow X \quad R: U \rightarrow W,
$$

such that

$$
C R=R_{c} Q_{P}^{T} .
$$

The operator $Q_{P}^{T}$ selects global coarse degrees of freedom in $U_{c}$ as linear combinations of global degrees of freedom; a global coarse degree of freedom is given by a row of $Q_{P}$. The operator $R$ (resp. $R_{c}$ ) restricts a vector of global (coarse) degrees of freedom into a vector of local (coarse) degrees of freedom. See Mandel and Sousedík [2007a] for more details.

\subsection{Multilevel BDDC}

The substructuring components (the domains, spaces and operators) from the previous section will be denoted by an additional subscript ${ }_{1}$, as $\Omega_{1}^{i}$, $i=1, \ldots N_{1}$, etc., and called level 1 . We will call the coarse problem in $\widetilde{W}_{\Pi 1}$ the level 2 problem. It has the same finite element structure as the original problem (1) on level 1, so we have $U_{2}=\widetilde{W}_{\Pi 1}$. Level 1 substructures are level 2 elements, level 1 coarse degrees of freedom are level 2 degrees of freedom. The shape functions on level 2 are the coarse basis functions in $\widetilde{W}_{\Pi 1}$, which are given by the conditions that the value of exactly one coarse degree of freedom is one and others are zero, and that they are energy minimal in $\widetilde{W}_{1}$. Note that the resulting shape functions on level 2 are in general discontinuous between level 2 elements. Level 2 elements are then agglomerated into nonoverlapping level 2 substructures, etc. Level $\ell$ elements are level $\ell-1$ substructures, and the level $\ell$ substructures are agglomerates of level $\ell$ elements. Level $\ell$ substructures are denoted by $\Omega_{\ell}^{i}$, and they are assumed to form a quasiuniform conforming triangulation with characteristic substructure size $H_{\ell}$. The degrees of freedom of level $\ell$ elements are given by level $\ell-1$ coarse degrees of freedom, and shape functions on level $\ell$ are determined by minimization of energy on each level $\ell-1$ substructure separately, so $U_{\ell}=\widetilde{W}_{\Pi, \ell-1}$. The averaging operators on level $\ell, E_{\ell}: \widetilde{W}_{\ell} \rightarrow U_{\ell}$, are defined by averaging of the values of level $\ell$ degrees of freedom between level $\ell$ substructures $\Omega_{\ell}^{i}$. The space $U_{I \ell}$ consists of functions in $U_{\ell}$ that are zero on the boundaries of all level $\ell$ substructures, and $P_{\ell}: U_{\ell} \rightarrow U_{I \ell}$ is the $a$-orthogonal projection in $U_{\ell}$ onto $U_{I \ell}$. For convenience, let $\Omega_{0}^{i}$ be the original finite elements, $H_{0}=h$. 
Algorithm 1 (Multilevel BDDC, Mandel et al. [2008], Algorithm 17) Define the preconditioner $r_{1} \in U_{1}^{\prime} \longmapsto u_{1} \in U_{1}$ as follows:

for $\ell=1, \ldots, L-1$,

Compute interior pre-correction on level $\ell$,

$$
u_{I \ell} \in U_{I \ell}: a\left(u_{I \ell}, z_{I \ell}\right)=\left\langle r_{\ell}, z_{I \ell}\right\rangle, \quad \forall z_{I \ell} \in U_{I \ell} .
$$

Get updated residual on level $\ell$,

$$
r_{B \ell} \in U_{\ell}, \quad\left\langle r_{B \ell}, v_{\ell}\right\rangle=\left\langle r_{\ell}, v_{\ell}\right\rangle-a\left(u_{I \ell}, v_{\ell}\right), \quad \forall v_{\ell} \in U_{\ell} .
$$

Find the substructure correction on level $\ell$,

$$
w_{\Delta \ell} \in W_{\Delta \ell}: a\left(w_{\Delta \ell}, z_{\Delta \ell}\right)=\left\langle r_{B \ell}, E_{\ell} z_{\Delta \ell}\right\rangle, \quad \forall z_{\Delta \ell} \in W_{\Delta \ell} .
$$

Formulate the coarse problem on level $\ell$,

$$
w_{\Pi \ell} \in W_{\Pi \ell}: a\left(w_{\Pi \ell}, z_{\Pi \ell}\right)=\left\langle r_{B \ell}, E_{\ell} z_{\Pi \ell}\right\rangle, \quad \forall z_{\Pi \ell} \in W_{\Pi \ell},
$$

If $\ell=L-1$, solve the coarse problem directly and set $u_{L}=w_{\Pi L-1}$, otherwise set up the right-hand side for level $\ell+1$,

$$
r_{\ell+1} \in \widetilde{W}_{\Pi \ell}^{\prime}, \quad\left\langle r_{\ell+1}, z_{\ell+1}\right\rangle=\left\langle r_{B \ell}, E_{\ell} z_{\ell+1}\right\rangle, \quad \forall z_{\ell+1} \in \widetilde{W}_{\Pi \ell}=U_{\ell+1},
$$

end.

for $\ell=L-1, \ldots, 1$,

Average the approximate corrections on substructure interfaces on level $\ell$,

$$
u_{B \ell}=E_{\ell}\left(w_{\Delta \ell}+u_{\ell+1}\right) .
$$

Compute the interior post-correction on level $\ell$,

$$
v_{I \ell} \in U_{I \ell}: a\left(v_{I \ell}, z_{I \ell}\right)=a\left(u_{B \ell}, z_{I \ell}\right), \quad \forall z_{I \ell} \in U_{I \ell} .
$$

Apply the combined corrections,

$$
u_{\ell}=u_{I \ell}+u_{B \ell}-v_{I \ell} .
$$

end.

A condition number bound follows, cf. [Mandel et al. [2008], Lemma 20].

Lemma 1. If for some $\omega_{\ell} \geq 1$, for all $\ell=1, \ldots, L-1$,

$$
\omega_{\ell}=\sup _{w_{\ell} \in\left(I-P_{\ell}\right) \widetilde{W}_{\ell}} J_{\ell}\left(w_{\ell}\right), \quad J_{\ell}\left(w_{\ell}\right)=\frac{\left\|\left(I-E_{\ell}\right) w_{\ell}\right\|_{a}^{2}}{\left\|w_{\ell}\right\|_{a}^{2}},
$$

then the multilevel BDDC preconditioner satisfies $\kappa \leq \omega=\prod_{k=1}^{L-1} \omega_{\ell}$. 


\section{Indicator of the Condition Number Bound}

As in Mandel and Sousedík [2007a], we propose as an indicator of the condition number the maximum of the bounds from Lemma 1 computed by considering on each level $\ell$ only one pair of adjacent substructures $s$ and $t$ at a time:

$$
\omega \approx \widetilde{\omega}=\Pi_{\ell=1}^{L-1} \max _{s t} \omega_{\ell}^{s t}, \quad \omega_{\ell}^{s t}=\sup _{w_{\ell}^{s t} \in\left(I-P_{\ell}^{s t}\right) \widetilde{W}_{\ell}^{s t}} J_{\ell}^{s t}\left(w_{\ell}^{s t}\right)
$$

where a pair of substructures is called adjacent if they share an edge, and the quantities with the superscript ${ }^{s t}$ are defined using the domain consisting of the level $\ell$ substructures $s$ and $t$ only.

The quantity $\widetilde{\omega}$ is called an indicator of the condition number bound.

Let $S_{\ell}^{s t}$ be the Schur complement operator associated with the bilinear form $a(\because \cdot)$ on the space $\left(I-P_{\ell}^{s t}\right) \widetilde{W}_{\ell}^{s t}$. The next theorem is [Mandel and Sousedík, 2006, Theorem 2] written in a way suitable for our purposes.

Theorem 2. Let $a>0, \Pi_{\ell}^{s t}$ be the orthogonal projection onto $\left(I-P_{\ell}^{s t}\right) \widetilde{W}_{\ell}^{s t}$, and $I-\bar{\Pi}_{\ell}^{s t}$ the orthogonal projection onto

$$
\operatorname{null}\left(\Pi_{\ell}^{s t} S_{\ell}^{s t} \Pi_{\ell}^{s t}+a\left(I-\Pi_{\ell}^{s t}\right)\right) .
$$

Then the stationary values $\omega_{\ell, 1}^{s t} \geq \omega_{\ell, 2}^{s t} \geq \ldots$ and the corresponding stationary vectors $w_{\ell, k}^{s t}$ of the Rayleigh quotient $J_{\ell}^{\text {st }}$ in (13) satisfy

$$
X_{\ell}^{s t} w_{\ell, k}^{s t}=\omega_{\ell, k}^{s t} Y_{\ell}^{s t} w_{\ell, k}^{s t}
$$

with $Y_{\ell}^{\text {st }}$ positive definite, where

$$
\begin{aligned}
X_{\ell}^{s t} & =\Pi_{\ell}^{s t}\left(I-E_{\ell}^{s t}\right)^{T} S_{\ell}^{s t}\left(I-E_{\ell}^{s t}\right) \Pi_{\ell}^{s t}, \\
Y_{\ell}^{s t} & =\left(\bar{\Pi}_{\ell}^{s t}\left(\Pi_{\ell}^{s t} S_{\ell}^{s t} \Pi_{\ell}^{s t}+a\left(I-\Pi_{\ell}^{s t}\right)\right) \bar{\Pi}_{\ell}^{s t}+a\left(I-\bar{\Pi}_{\ell}^{s t}\right)\right) .
\end{aligned}
$$

The eigenvalue problem (14) is obtained by projecting the gradient of the Rayleigh quotient $J_{\ell}^{s t}\left(w_{\ell}^{s t}\right)$ onto the complement in $\left(I-P_{\ell}^{s t}\right) \widetilde{W}_{\ell}^{s t}$ of the subspace, where its denominator is zero, in two steps. Both projections $\Pi_{\ell}^{s t}$ and $\bar{\Pi}_{\ell}^{s t}$ are computed by matrix algebra, which is straightforward to implement numerically. The projection $\Pi_{\ell}^{s t}$ projects onto null $C_{\ell}^{s t}\left(I-E_{\ell}^{s t}\right)$, and $I-\bar{\Pi}_{\ell}^{s t}$ projects onto a subspace of null $S_{\ell}^{s t}$, which can be easily constructed computationally if a matrix $Z_{\ell}^{s t}$ is given such that null $S_{\ell}^{s t} \subset$ range $Z_{\ell}^{s t}$. For this purpose, the rigid body modes are often available directly or they can be computed from the geometry of the finite element mesh. For levels $\ell>1$, we can use the matrix $Z_{\ell}^{s t}$ with columns consisting of coarse basis functions, because the span of the coarse basis functions contains the rigid body modes. In this way, we can reduce (14) to a symmetric eigenvalue problem, which is easier and more efficient to solve numerically. 


\section{Optimal Coarse Degrees of Freedom}

Writing $\widetilde{W}_{\ell}^{s t}=$ null $C_{\ell}^{s t}\left(I-E_{\ell}^{s t}\right)$ suggests how to add coarse degrees of freedom to decrease the value of indicator $\widetilde{\omega}$. The following theorem is an analogy to [Mandel and Sousedík, 2006, Theorem 3]. It follows immediately from the standard characterization of eigenvalues as minima and maxima of the Rayleigh quotient on subspaces spanned by eigenvectors, applied to (14).

Theorem 3. Suppose $n_{\ell}^{s t} \geq 0$ and the coarse dof selection matrix $C_{\ell}^{s t}\left(I-E_{\ell}^{s t}\right)$ is augmented by the rows $w_{\ell, k}^{s t T}\left(I-E_{\ell}^{s t}\right)^{T} S_{\ell}^{s t}\left(I-E_{\ell}^{s t}\right)$, where $w_{\ell, k}^{s t}$ are the eigenvectors from (14). Then $\omega_{\ell}^{s t}=\omega_{\ell, n_{\ell}^{s t}+1}^{s t}$, and $\omega_{\ell}^{s t} \geq \omega_{\ell, n_{\ell}^{s t}+1}^{s t}$ for any other augmentation by at most $n_{\ell}^{\text {st }}$ columns.

In particular, if $\omega_{\ell, n_{\ell}^{s t}+1}^{s t} \leq \tau$ for all pairs of adjacent substructures $s, t$ and for all levels $\ell=1, \ldots, L-1$, then $\widetilde{\omega} \leq \tau^{L-1}$.

Theorem 3 allows us to guarantee that the condition number indicator $\widetilde{\omega} \leq \tau^{L-1}$ for a given target value $\tau$, by adding the smallest possible number of coarse degrees of freedom.

The primal coarse space selection mechanism that corresponds to this augmentation can be explained as follows. Let us write the augmentation as

$$
c_{\ell, k}^{s t}=\left[c_{\ell, k}^{s} c_{\ell, k}^{t}\right]=w_{\ell, k}^{s t T}\left(I-E_{\ell}^{s t}\right)^{T} S_{\ell}^{s t}\left(I-E_{\ell}^{s t}\right),
$$

where $c_{\ell, k}^{s}$ and $c_{\ell, k}^{t}$ are blocks corresponding to substructures $s$ and $t$. It should be noted that the matrix $E_{\ell}^{s t}$ is constructed for a pair of substructures $s, t$ in such a way that, cf., e.g. [Mandel and Sousedík, 2007b, eq. (7)],

$$
B_{D, \ell}^{s t T} B_{\ell}^{s t}=I-E_{\ell}^{s t}
$$

where $B_{D, \ell}^{s t}$ and $B_{\ell}^{s t}$ are matrices known from the FETI-DP method. In particular, the entries of $B_{\ell}^{s t}$ are +1 for substructure $s$ and -1 for substructure $t$. This also relates our algorithm to the one from Mandel and Sousedík [2006], and Mandel and Sousedík [2007a]. Next, let us observe that, due to the application of $I-E_{\ell}^{s t}$, for the two blocks of $c_{\ell, k}^{s t}$ it holds that $c_{\ell, k}^{s}=-c_{\ell, k}^{t}$, i.e., for the two substructures the constraint weights have the same absolute values and opposite sign. Hence it is sufficient to consider only one of the two blocks, e.g., $c_{\ell, k}^{s}$. The augmentation of the global coarse degrees of freedom selection matrix $\left[Q_{P, \ell}, q_{k, \ell}\right]$ is constructed by adding a block of $k$ columns computed as

$$
q_{k, \ell}=R_{\ell}^{s T} c_{k}^{s T}
$$

Each column of $q_{k, \ell}$ defines a coarse degree of freedom associated with the interface of level $\ell$ substructures $s$ and $t$. Because $R_{\ell}^{s}$ is a $0-1$ matrix, it means that columns in $q_{k, \ell}$ are formed by a scattering of the entries in $c_{k}^{s T}$. 


\section{Adaptive-Multilevel BDDC in 2D}

We describe in more detail the implementation of the algorithm. It consists of two main steps: (i) setup, and (ii) the loop of preconditioned conjugate gradients with the Adaptive-Multilevel BDDC as a preconditioner. The setup was outlined in the previous section, and it can be summarized as follows:

Algorithm 4 Adding of coarse degrees of freedom in order to guarantee that the condition number indicator $\widetilde{\omega} \leq \tau^{L-1}$, for a given a target value $\tau$ :

for levels $\ell=1: L-1$,

Create substructures with roughly the same numbers of degrees of freedom, minimizing the number of "cuts" (use a graph partitioner, e.g., METIS 4.0 (Karypis and Kumar [1998]) with weights on both, vertices and edges).

Find a suitable set of initial constraints (corners in 2D), and set up the $B D D C$ structures for the adaptive algorithm.

\section{for all edges $\mathcal{E}_{\ell}$ on level $\ell$,}

Compute the largest local eigenvalues and corresponding eigenvectors, until the first $m^{s t}$ is found such that $\lambda_{m^{s t}}^{s t} \leq \tau$, put $k=1, \ldots, m^{s t}$.

Compute the constraint weights $c_{k}^{s t}=\left[\begin{array}{ll}c_{k}^{s} & c_{k}^{t}\end{array}\right]$ as

$$
c_{k}^{s t}=w_{k}^{s t T} \Pi_{\ell}^{I s t}\left(I-E_{\ell}^{s t}\right)^{T} S_{\ell}^{s t}\left(I-E_{\ell}^{s t}\right) \Pi_{\ell}^{I s t},
$$

where $\Pi_{\ell}^{I s t}$ is a projection constructed using the set of initial constraints.

Take one block, e.g., $c_{k}^{s}$ and keep nonzero weights for the edge $\mathcal{E}_{\ell}$.

Add to global coarse dofs selection matrix $Q_{P, \ell}$ the $k$ columns $q_{k, \ell}$ as

$$
q_{k, \ell}=R_{\ell}^{s T} c_{k}^{s T}
$$

end.

Setup the BDDC structures for level $\ell$ and check size of the coarse problem: if small enough, call this the level $L$ problem, factor it directly, and exit from the loop.

end.

We remark that the adaptive algorithm is significantly simpler and easier to implement compared to our previous algorithm from Mandel and Sousedík [2006], and Mandel and Sousedík [2007a]. The constraints in (15) are generated from the eigenvectors by the same function that evaluates the left hand side in (14). Then they are "torn" into two blocks, and entries of one of them, that correspond to a particular edge shared by substructures $s$ and $t$ on the level $\ell$, are scattered into additional columns of the matrix $Q_{P, \ell}$. 
The adaptive algorithm uses matrices and operators that are readily available in our implementation of the standard BDDC method (unlike in Mandel et al. [2009] this time with an explicit coarse space solve) with one exception: in order to satisfy the local partition of unity property, cf. [Mandel and Sousedík, 2007b, eq. (9)],

$$
E_{\ell}^{s t} R_{\ell}^{s t}=I
$$

we need to generate locally the weight matrices $E_{\ell}^{s t}$.

The substructures on higher levels are then treated as (coarse) elements with energy minimal basis functions. However, the number of added constraints is a-priori unknown. For this reason, the coarse elements must allow for variable number of nodes per element, and also for variable number of degrees of freedom per node. It is also essential to generate a sufficient number of corners as initial constraints, in particular to prevent rigid body motions between any pair of adjacent substructures. This topic has been addressed several times cf., e.g., a recent contribution by Burda et al. [2009].

Finally, we remark that instead of performing interior pre-correction and post-correction on level $\ell=1$, cf. eqs (4)-(5) and (10)-(11), we can benefit from reducing the problem to interfaces in the pre-processing step.

\section{Numerical Examples and Conclusion}

The adaptive-multilevel BDDC preconditioner was implemented in Matlab for the 2D linear elasticity problem (with $\lambda=1$, and $\mu=2$ ) on a square domain discretized by finite elements with 1182722 degrees of freedom. The domain was decomposed into 2304 subdomains on the second level and into 9 subdomains on the third-level. Such decomposition leads to the coarsening ratio $H_{\ell} / H_{\ell-1}=16$, with $\ell=1,2$. In order to test the adaptive selection of constraints, one single edge has been jagged on both decomposition levels, see Fig. 1. We have computed the eigenvalues and eigenvectors of (14) by setting up the matrices and using standard methods for the symmetric eigenvalue problem in Matlab, version 7.8.0.347 (R2009a).

In the first set of experiments, we have compared performance of the nonadaptive BDDC method with 2 and 3 decomposition levels. The results are presented in Tables 1 and 2. As expected from the theory the convergence of the algorithm deteriorates when additional levels are introduced.

In the next set of experiments, we have tested the adaptive algorithm for the two-level BDDC. The results are summarized in Table 6 . The algorithm performs consistently with our previous formulation in Mandel and Sousedík [2007a]. The eigenvalues associated with edges between substructures clearly distinguish between the single problematic edge and the others (Table 3). Adding the coarse dofs created from the associated eigenvectors according to Theorem 3 decreases the value of the condition number indicator $\widetilde{\omega}$ and improves convergence at the cost of increasing the number of coarse dofs. 


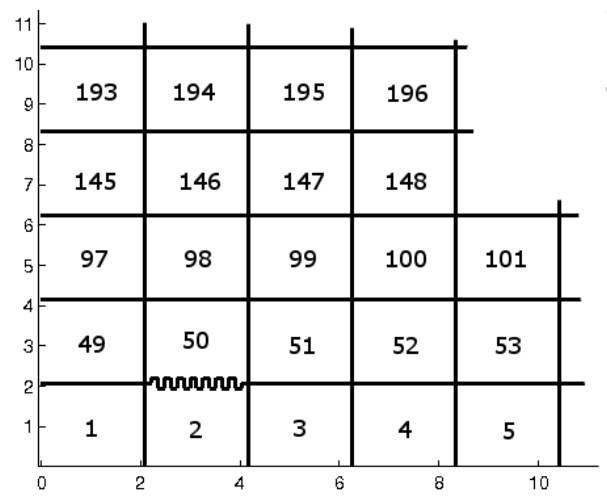

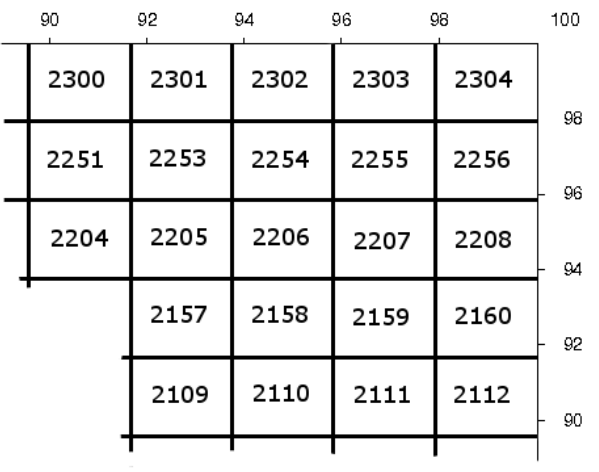

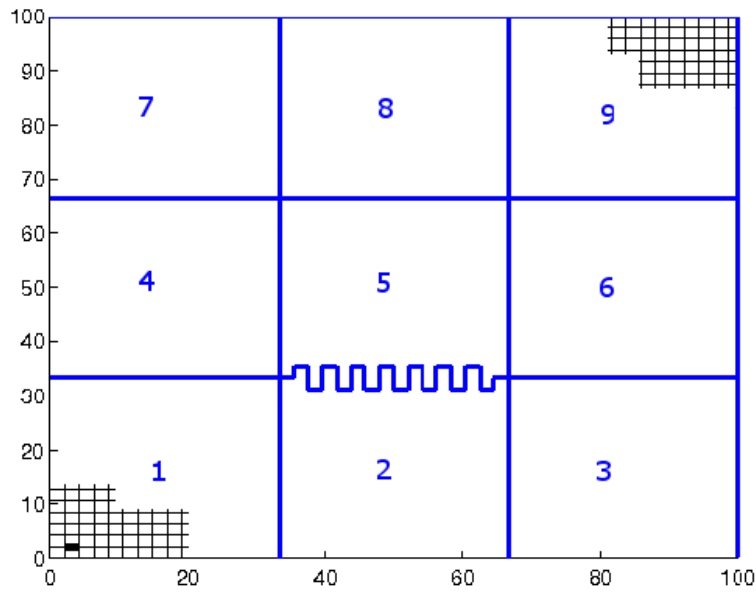

Fig. 1. The two-level decomposition into $48 \times 48(=2304)$ subdomains (top), and the decomposition into 9 subdomains for the three-level method (bottom); the jagged edge from the lower decomposition level is indicated here by a thick line. 
Table 1. Results for non-adaptive 2-level method. Constraints are corners, or corners and arithmetic averages over edges, denoted as c, c+e, resp. $N c$ is number of constraints, $\mathcal{C}$ is size of the coarse problem related to size of a subdomain problem, $\kappa$ is the condition number estimate, it is number of iterations (tol. $10^{-8}$ ).

\begin{tabular}{rrrrr}
\hline constraint & $N c$ & $\mathcal{C}$ & $\kappa$ & $i t$ \\
\hline c & 4794 & 9.3 & 18.41 & 43 \\
c+e & 13818 & 26.9 & 18.43 & 32
\end{tabular}

Table 2. Results for non-adaptive 3-level method. Headings are as in Table 1.

\begin{tabular}{rrrrr}
\hline constraint & $N c$ & $\mathcal{C}$ & $\kappa$ & $i t$ \\
\hline c & $4794+24$ & 1.0 & 67.5 & 74 \\
c+e & $13818+48$ & 3.0 & 97.7 & 70 \\
\hline
\end{tabular}

Table 3. Eigenvalues of the local problems for several pairs of subdomains $s$ and $t$ on the decomposition level $\ell=1$ (the jagged edge is between subdomains 2 and 50).

\begin{tabular}{rrrrrrrrrr}
\hline$s$ & $t$ & $\lambda_{s t, 1}$ & $\lambda_{s t, 2}$ & $\lambda_{s t, 3}$ & $\lambda_{s t, 4}$ & $\lambda_{s t, 5}$ & $\lambda_{s t, 6}$ & $\lambda_{s t, 7}$ & $\lambda_{s t, 8}$ \\
\hline 1 & 2 & 3.8 & 2.4 & 1.4 & 1.3 & 1.2 & 1.1 & 1.1 & 1.1 \\
1 & 49 & 6.0 & 3.5 & 2.7 & 1.4 & 1.3 & 1.1 & 1.1 & 1.1 \\
2 & 3 & 5.4 & 2.6 & 1.6 & 1.3 & 1.2 & 1.1 & 1.1 & 1.1 \\
2 & 50 & 24.3 & 18.4 & 18.3 & 16.7 & 16.7 & 14.7 & 13.5 & 13.1 \\
3 & 4 & 3.4 & 2.4 & 1.4 & 1.3 & 1.1 & 1.1 & 1.1 & 1.1 \\
3 & 51 & 7.4 & 4.6 & 3.7 & 1.7 & 1.4 & 1.3 & 1.2 & 1.1 \\
49 & 50 & 12.6 & 5.1 & 4.3 & 1.9 & 1.6 & 1.3 & 1.2 & 1.2 \\
50 & 51 & 8.7 & 4.8 & 3.9 & 1.8 & 1.5 & 1.3 & 1.2 & 1.2 \\
50 & 98 & 7.5 & 4.6 & 3.7 & 1.7 & 1.4 & 1.3 & 1.2 & 1.1 \\
\hline
\end{tabular}

Finally, we have tested the performance of the Adaptive-Multilevel BDDC for the model problem with three-level decomposition (Fig. 1). Because the number of coarse degrees of freedom depends on an a-priori chosen value of $\tau$ and the coarse basis functions on level $\ell$ become shape basis functions on level $\ell+1$, the solutions of local eigenvalue problems will depend on $\tau$ as well. This fact is illustrated by Table 4 for $\tau=2$, and by Table 5 for $\tau=10$ (the local eigenvalues for $\tau=3$ were essentially same as for $\tau=2$ ). Comparing the values in these two tables, we see that lower values of $\tau$ result in worse conditioning of the local eigenvalue problems on higher decomposition level. This immediately gives rise to a conjecture that it might not be desirable to decrease the values of $\tau$ arbitrarily low in order to achieve a better convergence of the method. On the other hand, for the model problem, comparing the convergence results for the two-level method (Table 6) with the three-level method (Table 7 ), we see that with the adaptive constraints we were able to achieve nearly the same convergence properties for the two methods. 
Table 4. Eigenvalues of the local problems for several pairs of subdomains $s, t$ on level $\ell=2$ with $\tau=2$ (the jagged edge is between subdomains 2 and 5 ).

\begin{tabular}{llrrrrrrrr}
\hline$s$ & $t$ & $\lambda_{s t, 1}$ & $\lambda_{s t, 2}$ & $\lambda_{s t, 3}$ & $\lambda_{s t, 4}$ & $\lambda_{s t, 5}$ & $\lambda_{s t, 6}$ & $\lambda_{s t, 7}$ & $\lambda_{s t, 8}$ \\
\hline 1 & 2 & 16.5 & 9.0 & 5.4 & 2.6 & 2.1 & 1.4 & 1.3 & 1.3 \\
1 & 4 & 6.5 & 4.7 & 1.9 & 1.7 & 1.3 & 1.2 & 1.2 & 1.1 \\
2 & 3 & 23.1 & 9.4 & 4.6 & 3.2 & 2.1 & 1.6 & 1.4 & 1.3 \\
2 & 5 & 84.3 & 61.4 & 61.4 & 55.9 & 55.8 & 49.3 & 48.0 & 46.9 \\
3 & 6 & 13.7 & 8.8 & 4.4 & 2.2 & 1.9 & 1.4 & 1.3 & 1.2 \\
4 & 7 & 6.5 & 4.7 & 1.9 & 1.7 & 1.3 & 1.2 & 1.2 & 1.1 \\
5 & 6 & 18.9 & 13.1 & 11.3 & 3.8 & 2.6 & 2.1 & 1.9 & 1.5 \\
5 & 8 & 17.3 & 12.9 & 10.8 & 3.6 & 2.3 & 2.0 & 1.8 & 1.4 \\
8 & 9 & 13.7 & 8.8 & 4.4 & 2.2 & 1.9 & 1.4 & 1.3 & 1.2 \\
\hline
\end{tabular}

Table 5. Eigenvalues of the local problems for several pairs of subdomains $s, t$ on level $\ell=2$ with $\tau=10$ (the jagged edge is between subdomains 2 and 5 ).

\begin{tabular}{|c|c|c|c|c|c|c|c|c|}
\hline & & & & & & & & $\lambda_{s t}$ \\
\hline & & & & & & & & \\
\hline & & & & & & & & \\
\hline 3 & & & & & & & & 1. \\
\hline 5 & & 17.2 & 13 & & 12 & & & 10 \\
\hline & & & & & & & & 1. \\
\hline & & & & & & & & 1. \\
\hline & & & & & & & & 1. \\
\hline & & & & & & & & 1.2 \\
\hline 9 & 6.1 & 4.2 & 2.5 & 1.5 & 1.3 & 1.2 & 1.1 & 1. \\
\hline
\end{tabular}

Table 6. Results for the adaptive 2-level method. Headings are same as in Table 1, and $\tau$ is the condition number target, $\widetilde{\omega}$ is the condition number indicator.

\begin{tabular}{rrrrrr}
\hline$\tau$ & $N c$ & $\mathcal{C}$ & $\widetilde{\omega}$ & $\kappa$ & $i t$ \\
\hline$\infty(=\mathrm{c})$ & 4794 & 9.3 & - & 18.41 & 43 \\
10 & 4805 & 9.4 & 8.67 & 8.34 & 34 \\
3 & 18110 & 35.3 & 2.67 & 2.44 & 15 \\
2 & 18305 & 35.7 & 1.97 & 1.97 & 13 \\
\hline
\end{tabular}

Table 7. Results for the adaptive 3 -level method. Headings are as in Table 6 , but the threshold $\tau$ is now used on each of the two decomposition levels and so $\widetilde{\omega} \leq \tau^{2}$.

\begin{tabular}{rrccrr}
\hline$\tau$ & $N c$ & $\mathcal{C}$ & $\widetilde{\omega}$ & $\kappa$ & $i t$ \\
\hline$\infty(=\mathrm{c})$ & $4794+24$ & 1.0 & - & 67.5 & 74 \\
10 & $4805+34$ & 1.0 & 84.97 & 37.42 & 60 \\
3 & $18110+93$ & 3.9 & 7.88 & 3.11 & 19 \\
2 & $18305+117$ & 4.0 & 3.84 & 2.28 & 15 \\
\hline
\end{tabular}




\section{References}

P. Burda, M. Čertíková, J. Damašek, A. Novotný, and J. Šístek. Selection of corners for the BDDC method. Submitted to Math. Comput. Simulation, 2009.

Clark R. Dohrmann. A preconditioner for substructuring based on constrained energy minimization. SIAM J. Sci. Comput., 25(1):246-258, 2003.

George Karypis and Vipin Kumar. METIS: A software package for partitioning unstructured graphs, partitioning meshes, and computing fill-reducing orderings of sparse matrices, version 4.0. Technical report, Department of Computer Science, University of Minnesota, 1998.

Axel Klawonn and Oliver Rheinbach. Inexact FETI-DP methods. International Journal for Numerical Methods in Engineering, 69(2):284-307, 2007.

Axel Klawonn and Oliver Rheinbach. A hybrid approach to 3-level FETI. PAMM, 8(1):10841-10843, 2008.

Axel Klawonn, Oliver Rheinbach, and Olof B. Widlund. An analysis of a FETI-DP algorithm on irregular subdomains in the plane. SIAM J. Numer. Anal., 46(5):2484-2504, 2008.

Jan Mandel, Clark R. Dohrmann, and Radek Tezaur. An algebraic theory for primal and dual substructuring methods by constraints. Appl. Numer. Math., 54(2):167-193, 2005.

Jan Mandel and Bedřich Sousedík. Adaptive coarse space selection in the BDDC and the FETI-DP iterative substructuring methods: Optimal face degrees of freedom. In Olof B. Widlund and David E. Keyes, editors, Domain Decomposition Methods in Science and Engineering XVI, Lecture Notes in Computational Science and Engineering, vol. 55, pages 421-428. Springer-Verlag, 2006.

Jan Mandel and Bedřich Sousedík. Adaptive selection of face coarse degrees of freedom in the BDDC and the FETI-DP iterative substructuring methods. Comput. Methods Appl. Mech. Engrg., 196(8):1389-1399, 2007a.

Jan Mandel and Bedřich Sousedík. BDDC and FETI-DP under minimalist assumptions. Computing, 81:269-280, 2007b.

Jan Mandel, Bedřich Sousedík, and Clark R. Dohrmann. Multispace and Multilevel BDDC. Computing, 83(2-3):55-85, 2008.

Jan Mandel, Bedřich Sousedík, and Jakub Šístek. Adaptive BDDC in three dimensions. Submitted to Math. Comput. Simulation, 2009.

Bedřich Sousedík. Adaptive-Multilevel BDDC. PhD thesis, University of Colorado Denver, Department of Mathematical and Statistical Sciences, 2010.

Xuemin Tu. Three-level BDDC in three dimensions. SIAM J. Sci. Comput., 29(4):1759-1780, 2007a.

Xuemin Tu. Three-level BDDC in two dimensions. Internat. J. Numer. Methods Engrg., 69(1):33-59, 2007b.

Xuemin Tu. A three-level BDDC algorithm for saddle point problems. submitted to Numer. Math., 2008. 